\title{
Structure From Motion Using Sequential Monte Carlo Methods *
}

\author{
Gang Qian and Rama Chellappa \\ Center for Automation Research and \\ Dept. of Electrical and Computer Engineering \\ University of Maryland \\ College Park, MD 20742
}

\begin{abstract}
In this paper, the structure from motion (SfM) problem is addressed using sequential Monte Carlo methods. A new SfM algorithm based on random sampling is derived to estimate the posterior distributions of camera motion and scene structure for the perspective projection camera model. Experimental results show that challenging issues in solving the structure from motion problem including errors in feature tracking, feature occlusion, motion/structure ambiguity, processing mixed-domain sequences and handling mismatched features can be well modeled and effectively addressed using the proposed method.
\end{abstract}

\section{Introduction}

In general, structure from motion (SfM) refers to the set of problems for recovering the 3D (stationary/dynamic) scene structure and sensor motion trajectory given a group of $2 \mathrm{D}$ (monocular/stereo) image frames obtained from an (calibrated/uncalibrated) optical camera. In this paper, we will focus on SfM using image frames captured by a monocular calibrated camera (i.e. the principal point and the field of view (FOV) of the camera are assumed to be known.)

The SfM problem has been a very active research area in computer vision since early eighties when Longuet-Higgins published the famous "eight point" algorithm [1]. The past two decades have witnessed a multitude of methods for solving the SfM problem using various camera models, observation sources, token types. Reviews and comparison of different SfM methods can be found in [2, 3]. Although many algorithms have been developed, few of them give satisfactory performance in real applications. Researchers are still searching for robust SfM algorithms. To develop an SfM method that performs well in practice, one must consider the following issues: (1) observation noise (noise present in token correspondences or in computing optical flow), (2) feature occlusion, (3) motion/structure recovery ambiguities, (4) mixture of image sequences with both small and

\footnotetext{
- Partially supported by the Office of Naval Research under the grant N00014-011-0265.
}

large baselines and (5) mismatched token and/or independent moving objects in the observed image frames. Being able to handle these issues is critical for producing practical SfM algorithms. Although recently, elegant methods have been reported in $[4,5]$, more progress needs to be made in addressing the issues raised above.

In this paper, we focus our efforts on developing robust statistical SfM method using noisy sparse feature correspondences from calibrated video sequences under perspective projection. Since a moving camera can be viewed as a dynamic system with its motion characterized by state parameters, a state space model can be used to describe the camera motion. However, due to perspective projection, the observation equation of the dynamic system is nonlinear. Although nonlinear filtering techniques such as the extended Kalman filter and its variants $[6,7]$ have been applied to solve the SfM problem, the results are not satisfactory. Recently, Sequential Monte Carlo (SMC) methods have received more attention for estimation, prediction, filtering and smoothing of nonlinear/non-Gaussian state space models. Several SMC methods have been proposed in various scenarios. Isard and Blake developed the Condensation algorithm [8] for shape and contour tracking. The Monte Carlo filter proposed by Kitagawa [9] can be viewed as a generalized algorithm dealing with the state estimation problem for nonlinear/non-Gaussian dynamic system. The particle filter (or bootstrap filter) [10] is also a similar variant of the SMC method. What is worthy of note is that a general framework for using the SMC methods in dynamic systems has been provided by Liu and Chen [11] and some of the above methods could be interpreted as special cases of the general sequential importance sampling framework.

In the SfM problem, both sensor motion and scene structure are estimated. In our approach, the sensor motion itself is estimated first using an SMC method based on the epipolar constraint and then the scene structure is recovered using the motion estimates. Recently, Markov chain Monte Carlo (MCMC) was used in the SfM problem [5] by Forsyth, Ioffe and Haddon. In [5], the approach followed the spirit of the work proposed by Tomasi and Kanade [12]. Instead of using 
the factorization method in [12], the hybrid MCMC method is used to locate the mode of the posterior distribution of the parameters of interest (rotation, shape and validity matrices). It was shown in [5] that the MCMC method is capable of selecting valid feature points for object shape reconstruction and moving object segmentation. However, since the method is a batch algorithm and a large dimensional parameter estimation problem is being solved, the procedure is time consuming. Although it is possible to develop a similar algorithm using the perspective projection model that is more suitable for practical applications, convergence problems and efficiency: of the resulting algorithm may limit its usefulness.

In this paper, we develop a recursive algorithm for finding the posterior distribution of the sensor motion parameters using the perspective projection camera model and the sequential importance sampling (SIS) technique [11]. The structure of the scene can be subsequently reconstructed. When the scene is dynamic, the algorithm can also detect points on the background. By finding the posterior distribution of motion and scene structure parameters, a much clearer picture of the structure of solution space can be obtained. Through this, not only good estimates can be found, but the uncertainty of the estimates can also be characterized. We show that the SfM algorithm using SIS is capable of handling the challenges mentioned above.

\section{Bayesian motion estimation}

To solve the SfM problem is to find the optimal estimates for sensor motion, scene geometry and segmentation which can provide the best interpretation of the observations using criterion such as the maximum posterior (MAP). In this paper, the posterior distribution of the parameters, Prob(parameters/observation), is approximated using random sampling methods. In this section, we first introduce the SIS technique. We then formulate the SfM problem using a state space model and then develop an SIS algorithm for finding an approximation to the posterior distribution of the state parameters.

\subsection{Sequential importance sampling}

The SIS method is a recently proposed technique for approximating the posterior distribution of the state parameters for a dynamic system [11]. Usually, the state space model of a dynamic system is described by observation and state equations. If the measurement is denoted by $\mathbf{y}_{t}$ and the state parameter by $\mathbf{x}_{t}$, essentially, the observation equation provides the conditional distribution of the observation given the state, $f_{t}\left(\mathbf{y}_{t} \mid \mathbf{x}_{t}\right)$. Similarly, the state equation gives the Markov transition distribution from time $t$ to the next time, $q_{t}\left(\mathbf{x}_{t+1} \mid \mathbf{x}_{t}\right)$. The goal is to find the posterior distribution of the states $\left(\mathbf{x}_{1}, \mathbf{x}_{2}, \cdots, \mathbf{x}_{t}\right)$ given all the available observations up to $t, \pi_{t}\left(\mathcal{X}_{t}\right)=P\left(\mathcal{X}_{t} \mid \mathcal{Y}_{t}\right)$, where $\mathcal{X}_{t}=\left\{\mathbf{x}_{i}\right\}_{i=1}^{t}$ and $\mathcal{Y}_{t}=\left\{\mathbf{y}_{i}\right\}_{i=1}^{t}$. One way to represent an approximation to the posterior distribution is by a set of samples and their corresponding weights.

Definition [11] A random variable $X$ drawn from a distribution $g$ is said to be properly weighted by a weighting function $w(X)$ with respect to the distribution $\pi$ iffor any integrable function $h$,

$$
E_{g}\{h(X) w(X)\}=E_{\pi}\{h(X)\} .
$$

$A$ set of random draws and weights $\left(x^{(j)}, w^{(j)}\right), j=$ $1,2, \cdots$, is said to be properly weighted with respect to $\pi$ if

$$
\lim _{m \rightarrow \infty} \frac{\sum_{j=1}^{m} h\left(x^{(j)}\right) w^{(j)}}{\sum_{j=1}^{m} w^{(j)}}=E_{\pi} h(X)
$$

for any integrable function $h$.

Suppose $\left\{\mathcal{X}_{t}^{(j)}\right\}_{j=1}^{m}$ is a set of random samples properly weighted by the set of weights $\left\{w_{t}^{(j)}\right\}_{j=1}^{m}$ with respect to $\pi_{t}$ and let $g_{t+1}$ be a trial distribution. Then the SIS procedure obtains the random samples and weights recursively as follows.

SIS steps: for $j=1, \cdots, m$,

(A) Draw $X_{t+1}=\mathbf{x}_{t+1}^{(j)}$ from $g_{t+1}\left(\mathbf{x}_{t+1} \mid \mathcal{X}_{t}^{(j)}\right)$. Attach $\mathbf{x}_{t+1}^{(j)}$ to form $\mathcal{X}_{t+1}^{(j)}=\left(\mathcal{X}_{t}^{(j)}, \mathbf{x}_{t+1}^{(j)}\right)$.

(B) Compute the "incremental weight" $u_{t+1}$ by

$$
u_{t+1}^{(j)}=\frac{\pi_{t+1}\left(\mathcal{X}_{t+1}^{(j)}\right)}{\pi_{t}\left(\mathcal{X}_{t}^{(j)}\right) g_{t+1}\left(\mathbf{x}_{t+1} \mid \mathcal{X}_{t}^{(j)}\right)}
$$

and let $w_{t+1}^{(j)}=u_{t+1}^{(j)} w_{t}^{(j)}$.

It can be shown [11] that $\left\{\mathcal{X}_{t+1}^{(j)}, w_{t+1}^{(j)}\right\}_{j=1}^{m}$ is properly weighted with respect to $\pi_{t+1}$. Hence, the above SIS steps can be applied recursively to get the properly weighted set for future time instants when corresponding observations are available. It is not difficult to show that given the properly weighted samples $\left\{\mathcal{X}_{t}\right\}$ with respect to the joint posterior distribution $\pi_{t}\left(\mathcal{X}_{t}\right)$, the "marginal" samples formed by the components in $\left\{\mathcal{X}_{t}\right\}$ of $\mathbf{x}_{i}$ are properly weighted by the same set of weights corresponding to the marginal posterior distribution $\pi_{t}\left(\mathbf{x}_{i}\right)$. Once the properly weighted samples of the joint distribution are obtained, the marginal distributions are approximated by the "marginal" samples weighted by the same set of weights.

The choice of the trial distribution $g_{t+1}$ is very crucial in the SIS procedure since it directly affects the efficiency of the proposed SIS method. In our approach, we used $g_{t+1}$ as

$$
g_{t+1}\left(\mathbf{x}_{t+1} \mid \mathcal{X}_{t}\right)=q_{t+1}\left(\mathbf{x}_{t+1} \mid \mathbf{x}_{t}\right)
$$

due to the convenience it provides during computations and the satisfactory performance the associated SIS method gives for SfM estimation. It can be shown that in this case $u_{t+1} \propto f\left(\mathbf{y}_{t+1} \mid \mathbf{x}_{t+1}\right)$, which is the conditional probability density function of the observations at $t+1$ given the state sample $\mathbf{x}_{t+1}$ and is also known as the likelihood function of $\mathbf{x}_{t+1}$ since the observations are fixed. 


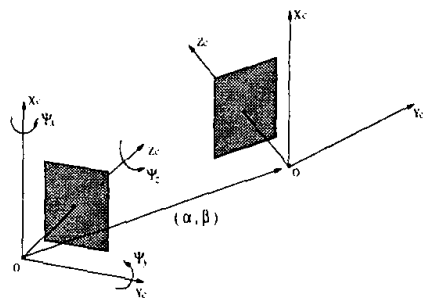

Figure 1: Camera motion model

\subsection{State space model for camera motion}

In the SfM problem, both sensor motion and scene structure are estimated. Multiple strategies exist and mainly there are two of them [3]. In one strategy, the sensor motion itself is first estimated using geometric constraints for rigid body motion such as the epipolar constraint and then the scene structure is recovered using the motion estimates. While in the other, structure and motion are estimated simultaneously. Since using the second strategy will result in a highdimensional state space (the dimension increases linearly with the number of feature points.) which is not favorable to the SIS procedure, we adopt the first strategy.

Parameterization of sensor motion Before discussing the parameterization of sensor motion, we introduce two 3D Euclidean coordinate systems used in our work. One coordinate system is attached to the camera and uses the center of projection of the camera as its origin. It is denoted by $C$. The $Z$ axis of $C$ is along the optical axis of the camera, with the positive half axis pointing to the scene being observed. The $X-Y$ plane of $C$ is perpendicular to $Z$ axis with $X$ and $Y$ axes parallel to the borders of the image plane. Also, $X-Y-Z$ axes of $C$ agree with the right-hand rule. The other coordinate system is a world inertial frame, denoted by $I . I$ is fixed on the ground. The coordinate axes of $I$ are configured in such a way that initially, $I$ and $C$ are coincident. When the camera moves, $C$ travels with the camera and $I$ stays at the initial position.

Five parameters are employed to describe the motion of the sensor at time $t$ relative to the world inertial frame $I$.

$$
\mathbf{x}_{t}=\left(\psi_{x}, \psi_{y}, \psi_{z}, \alpha, \beta\right)
$$

$\psi=\left(\psi_{x}, \psi_{y}, \psi_{z}\right)$ are the rotation angles of the camera about the coordinate axes of the world frame $I .(\alpha, \beta)$ are the elevation and azimuth angles of the camera translation direction, measured in the world system $I$. Let $T(\alpha, \beta)$ be the unit vector in the translation direction given by

$$
T(\alpha, \beta)=(\sin (\alpha) \cos (\beta), \sin (\alpha) \sin (\beta), \cos (\alpha))
$$

State space model Given the above motion parameterization, a state space model can be used to describe the behavior of a moving camera.

$$
\begin{aligned}
\mathbf{x}_{t+1} & =\mathbf{x}_{t}+n_{x} \\
\mathbf{y}_{t} & =\operatorname{Proj}\left(\mathbf{x}_{t}, \mathcal{S}_{t}\right)+n_{y}
\end{aligned}
$$

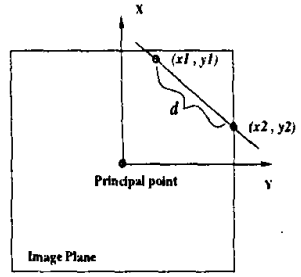

Figure 2: Epipolar line segment

where $\mathbf{x}_{t}$ is the state vector and $\mathbf{y}_{t}$ is the observation at time t. $\operatorname{Proj}(\cdot)$ denotes the perspective projection, a function of camera motion $\mathbf{x}_{t}$ and scene structure $\mathcal{S}_{t}, n_{x}$ denotes the dynamic noise in the system, describing the time varying property of the state vector. If no prior knowledge about motion is available, a random walk will be a suitable alternative for modeling the camera position.

\subsection{SIS for Bayesian motion estimation}

Based on the above state space model, we designed an SIS method for finding an approximation to the posterior distribution of the motion parameters. As mentioned above, the trial distribution in the SIS procedure used in our approach is chosen as $g_{t+1}\left(\mathbf{x}_{t+1} \mid \mathcal{X}_{t}^{\prime}\right)=q_{t+1}\left(\mathbf{x}_{t+1} \mid \mathbf{x}_{t}\right)$. Therefore, during SIS step (A), we will draw samples from the distribution of $\mathbf{x}_{t}+n_{x}$.

Likelihood function computation To derive the likelihood function, let us at first consider the case when there is only one single point $p$ that is observed. Assume that at time $t, p$ is projected to $(u, v)$ in the image plane. We assume that the noise in tracking a feature is normal with distribution $n_{y} \sim \mathcal{N}\left(0,\left(\begin{array}{cc}\sigma_{u} & 0 \\ 0 & \sigma_{v}\end{array}\right)\right)$. In the case of pure rotation, the likelihood function can be computed directly.

$$
f\left((u, v) \mid \mathbf{x}_{t}\right)=\frac{1}{2 \pi \sigma_{u} \sigma_{v}} \exp -\left\{\frac{\left(u-u^{\prime}\right)^{2}}{2 \sigma_{u}^{2}}+\frac{\left(v-v^{\prime}\right)^{2}}{2 \sigma_{v}^{2}}\right\}
$$

where $\left(u^{\prime}, v^{\prime}\right)$ gives the coordinates of the feature in image plane after camera rotation and it can be derived directly using (2). It is not difficult to see that in this pure rotation case, the scene structure $\mathcal{S}_{t}$ is not involved in the computation of $\left(u^{\prime}, v^{\prime}\right)$ given the pure rotation $\mathbf{x}_{t}$. However, the computation of $f\left(\mathbf{y}_{t} \mid \mathbf{x}_{t}\right)$ becomes difficult when the translation vector $T(\alpha, \beta)$ is nonzero. In this case, (3) can not be used directly since $\mathcal{S}_{t}$ is needed to compute $\left(u^{\prime}, v^{\prime}\right)$ when $T(\alpha, \beta) \neq 0$ while $\mathcal{S}_{t}$ is not represented in the state vector. In our approach, we used the conditional expectation over the structure to derive a formula for the likelihood function.

$$
f\left(\mathbf{y}_{t} \mid \mathbf{x}_{t}\right)=\int_{\mathbf{S}_{l}}^{\mathbf{s}_{u}} f\left(\mathbf{y}_{t} \mid \mathbf{x}_{t}, \mathcal{S}_{t}\right) p\left(\mathcal{S}_{t}\right) d \mathcal{S}_{t}
$$

where $\left[\mathbf{s}_{l}, \mathbf{s}_{u}\right]$ denotes the ranges of the feature depth such that all the features can be observed at both camera positions with positive feature depth values. However, the computation of the integral in (4) requires the knowledge of the prior 
distribution of the scene structure $\mathcal{S}_{t}$, which is unknown. To overcome this difficulty, the epipolar constraint is used. Recall that the epipolar constraint tells us that the perspective projections of a $3 \mathrm{D}$ point on the two image planes taken from different view points lie on their corresponding epipolar lines which are at the intersections of the two image planes and the epipolar plane. The epipolar plane is determined by that $3 \mathrm{D}$ point and two centers of projection (COP) of the camera at two positions. Also, given the image position of the point in the first view and camera motion, the epipolar line of the point in the second view can be easily determined. Note that

$$
E_{\mathcal{S}_{t}}\left\{f\left(\mathbf{y}_{t} \mid \mathbf{x}_{t}, \mathcal{S}_{t}\right)\right\}=E_{\mathcal{P}_{t}}\left\{f\left(\mathbf{y}_{t} \mid \mathbf{x}_{t}, \mathcal{P}_{t}\right)\right\}
$$

with $\mathcal{P}_{t}$ being the image position the feature points at time $t$. Although the prior distribution of $\mathcal{P}_{t}$ is not available either, a good guess of it is that $\mathcal{P}_{t}$ uniformly distributed on the pixel sites on their corresponding epipolar line segments. The epipolar line segments for each feature point can be easily determined using the eipoloar constraint and the positive depth constraint. Let $l$ denote the epipolar line segment of $p$ at $t$ and let $\left(x_{1}, y_{1}\right),\left(x_{2}, y_{2}\right)$ be the two terminal points of $l$. The locations of the two points are easy to find given the camera motion $\mathbf{x}_{t}$ and the image position of $p$ in the first frame using the positive depth constraint. Without loss of generality, let us assume that $\left|y_{2}-y_{1}\right| \geq\left|x_{2}-x_{1}\right|$ and $y_{2}>y_{1}$. Let $k=\frac{y_{2}-y_{1}}{x_{2}-x_{1}}$ be the slope of the epipolar line. The likelihood function of the motion parameter given one single point observation is given by

$$
f\left((u, v) \mid \mathbf{x}_{t}\right)=\frac{\sqrt{2 \pi} \lambda c}{2} \exp \left\{\frac{b^{2}}{2 a^{2} c^{2}}\right\}\left(\operatorname{erf}\left(f_{1}\right)-\operatorname{erf}\left(f_{2}\right)\right)
$$

where

$$
\left\{\begin{aligned}
f_{i} & =\frac{y_{i} a^{2}+b}{\sqrt{2} a c}, i=1,2 \\
\lambda & =\frac{1}{2 \pi c d} \exp \left\{-\left(\frac{\left(u-x_{1}\right)^{2}+2 k y_{1}\left(u-x_{1}+k y_{1}\right)}{2 \sigma_{u}^{2}}+\frac{v^{2}}{2 \sigma_{v}^{2}}\right)\right\} \\
d & =\sqrt{\left(y_{2}-y_{1}\right)^{2}+\left(x_{2}-x_{1}\right)^{2}} \\
c & =\sigma_{u} \sigma_{v} \\
b & =k\left(x_{1}-u-k y_{1}\right) \sigma_{v}^{2}-v \sigma_{u}^{2} \\
a & =\sqrt{\sigma_{v}^{2} k^{2}+\sigma_{u}^{2}}
\end{aligned}\right.
$$

and $\operatorname{er} f(\cdot)$ is the standard normal error function.

For a set of feature points, multiple likelihood functions can be applied under different situations. If all the points are "good" features in the sense that they are not on moving objects nor mismatched, then $f\left(\mathbf{y}_{t} \mid \mathbf{x}_{t}\right)$ is obtained by multiplying the individual likelihood function of each feature point.

$$
f\left(\mathbf{y}_{t} \mid \mathbf{x}_{t}\right)=\prod_{i=1}^{M} f\left(y_{t}^{(i)} \mid \mathbf{x}_{t}\right)=\prod_{i=1}^{M} f\left(\left(u_{t}^{(i)}, v_{t}^{(i)}\right) \mid \mathbf{x}_{t}\right)
$$

where $M$ is total number of observed feature points. In some cases, some of the points are known to be not "good" before hand. If the number of the "bad" points is less than half of the total number of tracked feature points, the following equation can be used to compute the likelihood function for the observations.

$$
f\left(\mathbf{y}_{t} \mid \mathbf{x}_{t}\right)=\prod_{i \in \mathcal{G}} f\left(y_{t}^{(i)} \mid \mathbf{x}_{t}\right)
$$

where $\mathcal{G}=\left\{i: f\left(y_{t}^{(i)} \mid \mathbf{x}_{t}\right) \geq \operatorname{median}\left(\left\{f\left(y_{t}^{(i)} \mid \mathbf{x}_{t}\right)\right\}_{i=1}^{M}, i=\right.\right.$ $1,2, \cdots, M\}$. The SIS method for Bayesian motion estimation is given as follows.

\section{SIS procedure}

1. Initialization. Draw samples of the motion parameters $\left\{\mathbf{x}_{0}^{(j)}\right\}_{j=1}^{m}$ from the initial distribution $\pi_{0} . \pi_{0}$ describes the distribution of motion parameters $\mathbf{x}_{0}$ before camera moves. The absence of camera motion does not imply that $\mathbf{x}_{0}=0$. Although the rotation angle vector $\psi$ and the translational vector are all zero, the translational angles can be uniformly distributed. Hence, in $\left\{\mathbf{x}_{0}^{(j)}\right\}$, the components of the rotation angles are all set to zero and the samples of $\alpha$ and $\beta$ are drawn from the uniform distribution in $[0, \pi]$ and $[0,2 \pi]$, respectively. Since all the samples are drawn from the exact distribution, equal weights are assigned to these samples.

For $t=1, \cdots, \tau$ :

2. Samples generation. Draw $\left\{\mathbf{x}_{t}^{(j)}\right\}_{j=1}^{m}$ from the distributions of $\left\{\mathbf{x}_{t-1}^{(j)}\right\}_{j=1}^{m}+n_{x}$. Since video sequences are used here as image sources instead of a set of image frames in arbitrary order, the following distribution can be used for $n_{x}$.

$$
\left\{\begin{aligned}
n_{\psi_{\iota}} & \sim \mathcal{N}\left(0, \sigma_{\iota}\right), \iota \in\{x, y, z\} \\
n_{\kappa} & \sim U\left(-\delta_{\kappa}, \delta_{\kappa}\right), \kappa \in\{\alpha, \beta\}
\end{aligned}\right.
$$

where $\sigma_{\iota}, \delta_{\alpha}$ and $\delta_{\beta}$ can be chosen as some positive numbers. At time $t=1$, some of the samples will be assigned to the group of pure rotation and the rest to general motion according to probability $P_{p r}$ which can be set based on the prior knowledge for the motion of camera. If it is not available, 0.5 can be a good guess. When $t>1$, some samples in the two groups are exchanged to accommodate the shift between pure rotation and general motion.

3. Weight computation and re-sampling. Compute the weights of the samples, $\left\{w_{t}^{(j)}\right\}$, using the observed feature correspondence according to (7) or (8) based on the problem at hand. The resulting samples and their corresponding weights $\left(\mathcal{X}_{t}^{(j)}, w_{t}^{(j)}\right)$ are properly weighted with respect to $\pi_{t}\left(\mathcal{X}_{t}\right)$. Re-sample the above samples.

By using the above properly weighted sample-weight sets for each time instant, the mean of motion parameters can be computed directly. Also since the sample-weight sequences after re-sampling approximates $\mathcal{X}_{t}$ in distribution, 
the MAP estimates of $\mathcal{X}_{t}$ can also be obtained by locating the modes of $\pi_{t}\left(\mathcal{X}_{t}\right)$.

\section{Estimation of depth distribution}

In this section, we show that the posterior distribution of the depth values of the feature points can also be approximated by using a set of samples drawn directly from the the target distribution. General sequences with both static and dynamic scenes are handled. Although some bad feature points (mismatched features or points on moving objects) can be segmented out using a similar SIS procedure which has been developed to detect moving targets, it can not detect points moving along epipolar lines. Hence, we do not use the scene rigidity constraint. The depth value of a feature point can be viewed as a random process with distribution $p_{t}(\mathbf{z})$. What we are interested in is the posterior distribution of $\mathbf{z}, p\left(\mathbf{z} \mid \mathcal{Y}_{\tau}\right)$ with $\mathcal{Y}_{\tau}=\left\{\mathbf{y}_{t}\right\}_{t \in[0, \tau\}}$ is the observation up to time $\tau$. If time index $t$ is also viewed as a random variable, $p\left(\mathbf{z} \mid \mathcal{Y}_{\tau}\right)$ is actually the marginal distribution of the joint distribution of $(\mathbf{z}, t)$.

$$
\begin{aligned}
p\left(\mathbf{z} \mid \mathcal{Y}_{\tau}\right) & =\int_{0}^{\tau} p\left(t, \mathbf{z}_{t} \mid \mathcal{Y}_{\tau}\right) d t \\
& =\int_{0}^{\tau} p\left(\mathbf{z}_{t} \mid \mathcal{Y}_{\tau}, t\right) p\left(t \mid \mathcal{Y}_{\tau}\right) d t \\
& =\int_{0}^{\tau} p\left(\mathbf{z}_{t} \mid \mathcal{Y}_{\tau}, t\right) p\left(t \mid \mathcal{Y}_{\tau}\right) d t
\end{aligned}
$$

where $p\left(t \mid \mathcal{Y}_{\tau}\right)$ is distribution of $t$ given that $t$ is in time interval $[0, \tau]$. Since we know that during the experiment of taking the video sequence each value of $t$ in $[0, \tau]$ is reached once and only once, $t$ can be viewed as a uniformly distributed random variable in $[0, \tau]$. Hence (12) can be rewritten as

$$
p\left(\mathbf{z} \mid \mathcal{Y}_{\tau}\right)=\frac{1}{\tau} \int_{0}^{\tau} p\left(\mathbf{z}_{t} \mid \mathcal{Y}_{\tau}, t\right) d t
$$

and in the case of discrete time, (13) becomes

$$
p\left(\mathbf{z} \mid \mathcal{Y}_{T}\right)=\frac{1}{T} \sum_{t=1}^{T} p\left(\mathbf{z}_{t} \mid \mathcal{Y}_{\tau}, t\right)
$$

where $T$ the number of frames observed up to time $\tau$.

We want to find the approximate depth distribution $p\left(\mathbf{z} \mid \mathcal{Y}_{T}\right)$ using the sequence of perspective projections of 3D feature points (their world coordinates might be timevarying) given the estimated posterior distribution of motion parameters at each frame time. We have the following theorems.

Theorem 1 Assume that $\mathcal{S}_{1}, \mathcal{S}_{2}, \cdots, \mathcal{S}_{k}$ is a series of sample sequences. $\mathcal{S}_{i}=\left\{x_{i}^{(j)}\right\}_{j=1}^{n}$ are properly weighted by normalized weights $\mathcal{W}_{i}=\left\{w_{i}^{(j)}\right\}_{j=1}^{n}$ with respect to distribution $\pi_{i}(x)$ with $n \gg 1$. If a distribution $\pi(x)$ satisfies

$$
\pi(x)=\sum_{i=1}^{k} a_{i} \pi_{i}(x)
$$

where $a_{i}>0$ for $i=1,2, \cdots, k$, then the collection of the above sample sequences $\left\{\mathcal{S}_{i}\right\}_{i=1}^{k}$ is a set of samples properly weighted by their respective weights $\left\{a_{i} \mathcal{W}_{i}\right\}_{i=1}^{k}$ with respect to $\pi(x)$.

Theorem 2 Given $\left\{\mathbf{y}_{0}, \mathbf{y}_{t}\right\}$ as two perspective views of a set of $3 D$ points, assuming that the translation vector is nonzero and that the smoothed posterior distribution of the camera motion parameter at time $t$ given $\mathcal{Y}_{\tau} \pi_{\tau}\left(\mathbf{x}_{t}\right)$, is approximated by a set properly weighted samples $\left\{\mathbf{x}^{(i)}\right\}_{i=1}^{k}$ and normalized weights $\left\{w^{(i)}\right\}_{i=1}^{k}$ and the prior depth distribution $\pi(\mathbf{z})$ is known, if $\mathcal{Z}_{t}$ and $\mathcal{W}_{t}$ are obtained from the following sampling method,

For each motion sample $\mathbf{x}^{(i)}, i=1, \cdots, k$

(A) Draw a set of samples $Z_{i}=\left\{\mathbf{z}_{i}^{(j)}\right\}$ around $\mathbf{z}^{*}$ from a trial distribution $g\left(\mathbf{z} \mid \mathbf{z}^{*}\right)$. Here $\mathbf{z}^{*}$ is called the maximum likelihood depth (MLD) and is given by

$$
\mathbf{z}^{*}=\arg \max _{\mathbf{z}} p\left(\mathbf{y}_{t} \mid \mathbf{z}, \mathbf{x}^{(i)}\right) \pi(\mathbf{z})
$$

(B) Compute the weights for the elements in $Z_{i}$ by

$$
w_{i}^{(j)}=\frac{p\left(\mathbf{y}_{t} \mid \mathbf{z}_{i}^{(j)}, \mathbf{x}^{(i)}\right) \pi\left(\mathbf{z}_{i}^{(j)}\right)}{g\left(\mathbf{z}_{i}^{(j)} \mid \mathbf{z}^{*}\right)} \propto \frac{p\left(\mathbf{z}_{i}^{(j)} \mid \mathbf{x}^{(i)}, \mathbf{y}_{t}\right)}{g\left(\mathbf{z}_{i}^{(j)} \mid \mathbf{z}^{*}\right)}
$$

and then normalize the weights by

$$
\tilde{w}_{i}^{(j)}=\frac{w_{i}^{(j)}}{\sum_{j} w_{i}^{(j)}}
$$

i.e. $Z_{i}$ is properly weighted by $W_{i}=\left\{\tilde{w}_{i}^{(j)}\right\}$ and (C) Let $\mathcal{Z}_{t}=\left\{Z_{i}\right\}_{i=1}^{k}$ and $\mathcal{W}_{t}=\left\{w^{(i)} W_{i}\right\}_{i=1}^{k}$.

Then $\mathcal{Z}_{t}$ is properly weighted by $\mathcal{W}_{t}$ with respect to the posterior depth distribution $\pi_{r}\left(\mathbf{z}_{t}\right)=p\left(\mathbf{z}_{t} \mid \mathcal{Y}_{T}\right)$.

A sketch of the proof:

$$
\begin{aligned}
p\left(\mathbf{z}_{t} \mid \mathcal{Y}_{\tau}\right) & =\int p\left(\mathbf{z}_{t}, \mathbf{x}_{t} \mid \mathcal{Y}_{\tau}\right) d \mathbf{x}_{t}=\int p\left(\mathbf{z}_{t} \mid \mathbf{x}_{t}, \mathcal{Y}_{\tau}\right) p\left(\mathbf{x}_{t} \mid \mathcal{Y}_{\tau}\right) d \mathbf{x}_{t} \\
& =\int p\left(\mathbf{z}_{t} \mid \mathbf{x}_{t}, \mathbf{y}_{t}\right) p\left(\mathbf{x}_{t} \mid \mathcal{Y}_{\tau}\right) d \mathbf{x}_{t}=E_{\pi_{\tau}\left(\mathbf{x}_{t}\right)} p\left(\mathbf{z}_{t} \mid \mathbf{x}_{t}, \mathbf{y}_{t}\right) \\
& =\lim _{k \rightarrow \infty} \frac{p\left(\mathbf{z}_{t} \mid \mathbf{x}_{t}, \mathbf{y}_{t}\right) w^{(i)}}{\sum_{i=1}^{k} w^{(i)}}
\end{aligned}
$$

Proposition 1 The distribution $p\left(\mathbf{z}_{t} \mid \mathbf{x}_{t}, \mathbf{y}_{t}\right)$ is proportional to $p\left(\mathbf{y}_{t} \mid \mathbf{x}_{t}, \mathbf{z}_{t}\right) \pi\left(\mathbf{z}_{t}\right)$.

Theorem 1 and Proposition 1 are not difficult to prove and the proofs are omitted here due to lack of space. Based on them, Theorem 2 can be easily justified using the above sketch of the proof. If the prior distribution of the depth is assumed to be uniform, the MLD $\mathbf{z}^{*}$ of the feature points can be computed as follows. Let $z$ be the depth value of $p$, which has image positions $y_{0}=\left(u_{0}, v_{0}\right)$ and $y=(u, v)$ in the first frame and the current frame at time $t$, respectively. Assume $\hat{\mathbf{x}}_{t}$ be a motion estimate between the two image frames found using the previously described SIS method. The least square 
criterion is applied to find the depth estimates at each time instant.

$$
\hat{z}_{t}=\arg \min _{z}\left\|y-\operatorname{Proj}\left(\hat{\mathbf{x}}_{t}, y_{0}, z\right)\right\|^{2}
$$

After some straightforward algebra, $\hat{z}_{t}$ is obtained as follows.

$$
\hat{z}_{t}=\frac{\left(t_{x} r_{z}-t_{z} r_{x}\right)\left(t_{z} u-t_{x}\right)+\left(t_{y} r_{z}-t_{z} r_{y}\right)\left(t_{z} v-t_{y}\right)}{\left(t_{x} r_{z}-t_{z} r_{x}\right)\left(r_{z} u-r_{x}\right)+\left(t_{y} r_{z}-t_{z} r_{y}\right)\left(r_{z} v-r_{y}\right)}
$$

where

$$
\left\{\begin{aligned}
\left(t_{x}, t_{y}, t_{z}\right)^{T} & =R(\psi) T(\alpha, \beta)^{T} \\
\left(r_{x}, r_{y}, r_{z}\right)^{T} & =R(\psi)\left(u_{0}, v_{0}, f\right)^{T}
\end{aligned}\right.
$$

and $f$ is the focal length in the unit of image plane width .

From Theorem 2, a set of properly weighted samples $\mathcal{Z}_{t}$ and associated weight $\mathcal{W}_{t}$ can be obtained with respect to $\pi_{\tau}\left(\mathbf{z}_{t}\right)$, the posterior distribution of the depth at time $t$ using the sampling method described. Furthermore, from Theorem 1 we know that $\left\{\mathcal{Z}_{t}\right\}_{t=1}^{\tau}$, the collection of the set of samples at each time instant is actually properly weighted by the collection of the associated weights $\left\{\mathcal{W}_{t}\right\}_{t=1}^{\tau}$ with respect to the posterior distribution of depth up to the current time $\tau$, namely $p\left(\mathbf{z} \mid \mathcal{Y}_{\tau}\right)$. Therefore, based on Theorem 2, a recursive algorithm has been developed to find an approximation to the posterior depth distribution.

\section{Recursive algorithm for depth distribution}

For each time instant $t=1,2, \cdots, \tau$,

(A) Compute the properly weighted samples $\left\{\mathbf{x}_{t}^{(i)}\right\}_{i=1}^{k}$ and their normalized weights $\left\{w_{t}^{(i)}\right\}_{i=1}^{k}$ with respect to $\pi_{\tau}\left(\mathbf{x}_{t}\right)$ using the above SIS procedure.

(B) For $i=1, \cdots, k$, generate $\left(Z_{i}, W_{i}\right)$ respect to each motion sample $\mathbf{x}_{t}^{(i)}$. The resulting samples $\mathcal{Z}_{t}=\left\{Z_{i}\right\}_{i=1}^{k}$ are properly weighted by $\mathcal{W}_{t}=\left\{w_{t}^{(i)} W_{i}\right\}_{i=1}^{N}$ with respect to $\pi_{\tau}\left(\mathbf{z}_{t}\right)$ (Theorem 2).

(D) Combine $\mathcal{Z}_{t}, \mathcal{W}_{t}$ for $t=1,2, \cdots, \tau$. The collection $\left\{\mathcal{Z}_{t}\right\}_{t=1}^{\tau}$ is a set of samples properly weighted by $\left\{\mathcal{W}_{t}\right\}_{t=1}^{\tau}$ with respect to the posterior distribution $p\left(\mathbf{z} \mid \mathcal{Y}_{\tau}\right)$ (Theorem $1)$.

Once the posterior distribution of the depth is approximated, the mean of the resulting distribution can be used as an estimate for depth values. This recursive algorithm requires the computation of $\pi_{\tau}\left(\mathbf{z}_{t}\right)$ at each time instant when new observations are available. Actually, this is not necessary. If $\pi_{t}\left(\mathbf{x}_{t}\right)$, the posterior distribution of the motion parameters given the observations up to time $t$ starts to converge after $t_{0}$, namely $\pi_{t}\left(\mathbf{x}_{t}\right)$ becomes very close to $\pi_{\tau}\left(\mathbf{x}_{t}\right)$, the smoothed posterior distribution of $\mathbf{x}_{t}$ given the entire observations $\mathcal{Y}_{\tau}$, the samples and weights computed in the above SIS procedure at time instant $t>t_{0}$ can be preserved for use at future time instants. Therefore, when new observations $\mathbf{y}_{\tau+1}$ become available, only $\mathcal{Z}_{\tau+1}$ and $\mathcal{W}_{\tau+1}$ need to be computed and then the collection of the new samples, weights and the preserved ones $\left(\left\{\mathcal{Z}_{t}\right\}_{t=1}^{\tau+1},\left\{\mathcal{W}_{t}\right\}_{t=1}^{\tau}\right)$ are a pair of sample-weight properly weighted with respect to $p\left(\mathbf{z} \mid \mathcal{Y}_{\tau+1}\right)$.

If the prior knowledge about the scene structure tells us that the scene is rigid, we have the following theorem for computing the posterior distribution of the depth.

Theorem 3 Given $\mathcal{Y}_{\tau}$ as a series of perspective views of a set of rigid $3 D$ points taken at time $t=1,2, \cdots, \tau$, if $\mathcal{Z}$ and $\mathcal{W}$ are obtained in the following way,

For each motion sample $\mathcal{X}^{(i)}, i=1, \cdots, k$

(A) Draw $Z_{i}=\left\{\mathbf{z}_{i}^{(j)}\right\}$ around $\mathbf{z}^{*}$ from a trial distribution $g\left(\mathbf{z} \mid \mathbf{z}^{*}\right) . \mathbf{z}^{*}$ is given by

$$
\mathbf{z}^{*}=\arg \max _{\mathbf{z}} p\left(\mathcal{Y}_{\tau} \mid \mathbf{z}, \mathcal{X}^{(i)}\right) \pi(\mathbf{z})
$$

(B) Compute the weight for the elements in $Z_{i}$ by

$$
w_{i}^{(j)}=\frac{p\left(\mathcal{Y}_{\tau} \mid \mathbf{z}_{i}^{(j)}, \mathcal{X}^{(i)}\right) \pi\left(\mathbf{z}_{i}^{(j)}\right)}{g\left(\mathbf{z}_{i}^{(j)} \mid \mathbf{z}^{*}\right)}
$$

and then normalize the weights by $\tilde{w}_{i}^{(j)}=\frac{w_{i}^{(j)}}{\sum_{j} w_{i}^{(j)}}$ Hence, $Z_{i}$ is properly weighted by $W_{i}=\left\{\tilde{w}_{i}^{(j)}\right\}$.

(C) Let $\mathcal{Z}=\left\{Z_{i}\right\}_{i=1}^{k}$ and $\mathcal{W}=\left\{w^{(i)} W_{i}\right\}_{i=1}^{k}$.

Then $\mathcal{Z}$ is properly weighted by $\mathcal{W}$ with respect to the posterior depth distribution $\pi_{\tau}(\mathbf{z})=p\left(\mathbf{z} \mid \mathcal{Y}_{\tau}\right)$.

Theorem 3 can be proved easily and a recursive algorithm similar to the above algorithm has been developed.

\section{Experiments}

By using the above SIS methods, challenging issues in SfM problem such as occlusion, SfM ambiguity, mixed-domain sequence processing, and mismatched feature points can be elegantly handled.

\subsection{A nominal case study}

Before we present real image results, we will first show some results using a synthetic sequence with feature trajectories corrupted by additive white Gaussian noise with standard deviation 0.5 pixel. The motion distributions are shown in Figure 3 . The ground truth of the motion parameters are indicated by solid lines. Figures $(a, b, c)$ in the first row of Figure 3 show the distribution of the rotational angles $\psi_{x}, \psi_{y}$ and $\psi_{z}$, respectively. The remaining two figures give the distribution of the translational angles $\alpha$ and $\beta$. In each figure, the distribution of the corresponding motion parameter at each time instant is shown from the top of the figure to the bottom with $Y$ index being the time instant and $X$ index being the range of each motion parameter. For $\left(\psi_{x}, \psi_{y}, \psi_{z}\right)$, they are in $[-\pi, \pi] . \alpha$ is in $[0, \pi]$ and $\beta$ ranges in $[0,2 \pi]$. All the other motion distribution results in this paper can be interpreted in the same way. We can see that the distributions of motion parameters have peaks very close to 

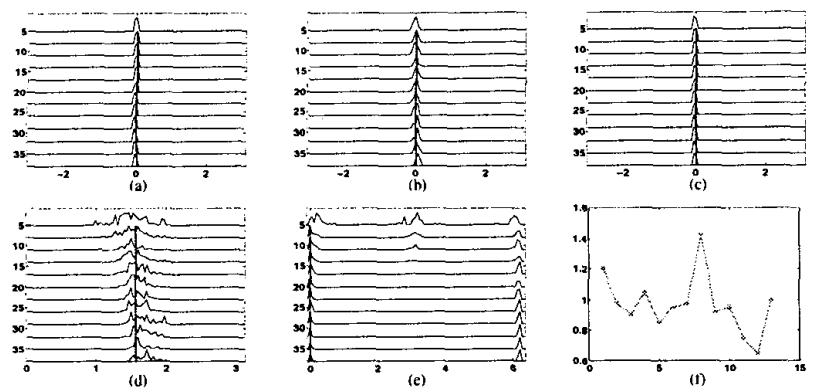

Figure 3: Results in the nominal case. (a)-(e) are the posterior distribution of the camera motion. (f) shows the grouth truth and MMSE estimtes of the feature depth.

the ground-truth. In Figure 3(e), two modes can be seen near 0 and $2 \pi$. They actually form one mode near 0 due to the periodic property of $\beta$, the azimuth angle of the camera translation direction. Figure 3(f) shows the ground truth and the estimates of the feature depth. Since they are very close, it is difficult to distinguish one from another.

\subsection{Feature occlusion}

During tracking feature points through the video sequence, some of the feature points might be occluded by some objects over a certain number of frames. This makes the occluded feature points unobservable during that period of time. They might become visible in subsequent frames. In Tomasi and Kanade's factorization method [12], the image positions of the occluded feature points are interpolated using both past and future positions of the respective feature point. In our approach, feature occlusion does not affect the basic theory of our algorithm. At each time instant, the occluded feature points are just ignored when the weights are computed and only visible feature points are used as observations. The reduction in the number of observed feature points might make the motion estimation less accurate in the sense that the resulting posterior distribution has a larger variance than when more observations are used.

\subsection{Motion and structure ambiguity}

The ambiguity present in the motion and structure recovery has been noted and found to be inherent [13]. Although the ambiguity in SfM can be reduced by using inertial rate data obtained from an inertial sensor [14], inertial sensors are not widely used yet and not many sequences are captured using cameras with inertial sensors. Given a set of feature correspondence tracked through such a sequence, two questions can be asked. The first one is whether the SfM ambiguity exists for this sequence and the second one is if it exists, what are those admissible solutions? When using the SIS method, answers to both questions can be found by looking at $\hat{\pi}_{t}\left(\mathbf{x}_{t}\right)$, the approximate posterior distribution of the motion parameters. Recall that the re-sampled properly weighted samples approach $\hat{\pi}_{t}\left(\mathbf{x}_{t}\right)$ asymptotically. For observations with SfM ambiguity, multiple modes can be found in $\hat{\pi}_{t}\left(\mathbf{x}_{t}\right)$. This an-
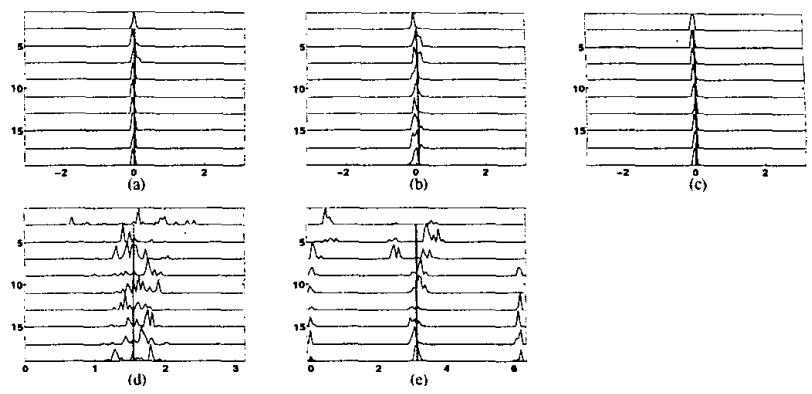

Figure 4: The posterior distribution of the motion parameters in the structure/motion ambiguity case.
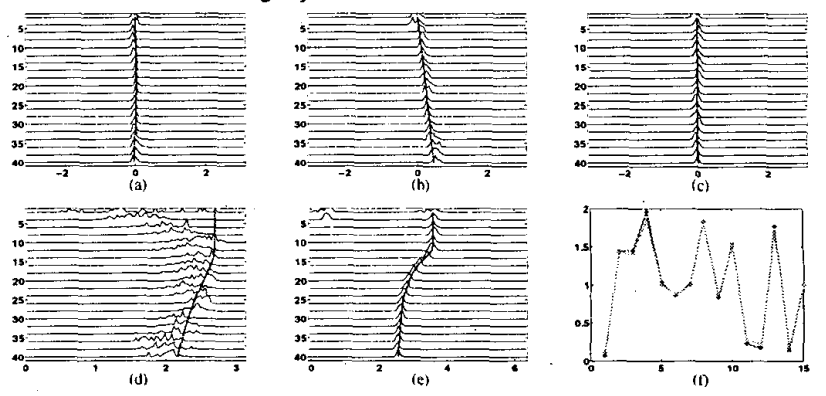

Figure 5: Results of processing a mixed-domain sequence. (a)(e) are the posterior distribution of the camera motion. (f) shows the grouth truth and MMSE estimtes of the feature depth.

swers the first question. Admissible solutions can be found by locating the modes of $\hat{\pi}_{t}\left(\mathbf{x}_{t}\right)$ in the solution space. The results shown in Figure 4 are obtained using an ambiguous sequence. We could observe in Figure 4 (e) that at least two different peaks exist in the distribution of the translational direction angle $\beta$, one is near 0 and the other is near $\pi$.

\subsection{Mixed-domain sequence processing}

Processing of mixed-domain sequences has been discussed by Oliensis [3] as a challenge for the SfM algorithms. Due to the assumption about sensor translation (small or large) made by the researchers when the SfM algorithms are designed, many existing SfM algorithms do not work well for mixed-domain sequences. In our approach, since no assumptions about translation are made, mixed-domain sequences can also be correctly processed. The results of processing a mixed-domain sequence are shown in Figure 5.

\subsection{Experiments using real images}
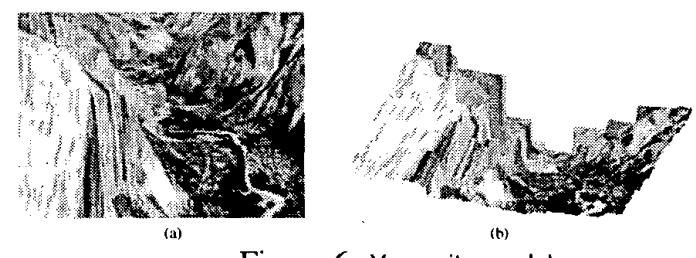

Figure 6: Yosemite model

Real image sequences have been used to test the proposed algorithm. Feature points were detected and tracked through 


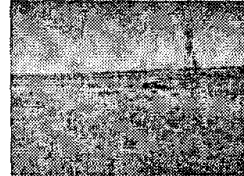

(a)

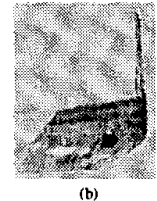

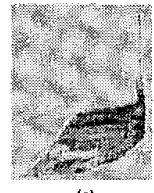

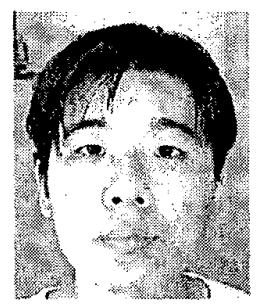

(a)

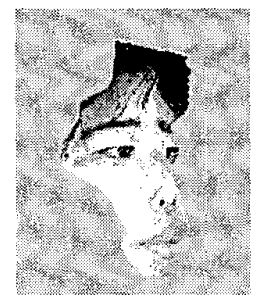

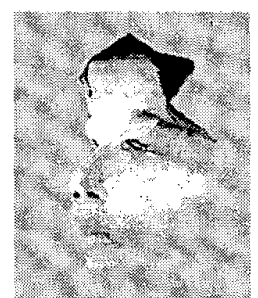

(b)

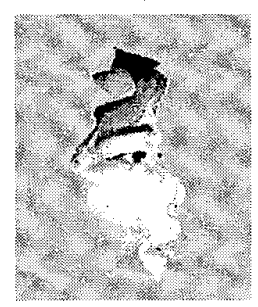

Figure 8: 3D face model

the sequences by using the well known KLT tracker [15]. The first experiment results are obtained by using the well known Yosemite sequence. The reconstructed 3D model is shown in Figure 6(b). The depth variation in the valley can be clearly observed in the reconstructed model. In the second example, an outdoor sequence is used. In the scene, there is a moving vehicle and some of the feature points are located on the moving vehicle. Figure 7(a) shows one of the frame from the original sequence and Figures $7(b, c)$ give the reconstructed 3D model. We can clearly see the $3 \mathrm{D}$ structure of the tower and the plane formed by the grass land. In the third example, a 3D face model is reconstructed from a face sequence. Since the proposed method is feature-based, the depth values of a set of feature points are first computed using the proposed approach and then the depth field of the entire object is obtained simply by using the interpolation function griddata in MATLAB 5.3.1. Figures 8(a) and (b,c,d) show the intensity map of the face and the reconstructed face model from different view points, respectively.

\section{Conclusion}

An SIS based SfM algorithm is presented. The posterior distribution of the camera motion and scene structure are approximated by sample and weight set. Experimental results using both synthetic and real images show that the proposed method is capable of dealing with the difficulties met in the SfM problem due to errors in feature tracking, mismatched features, feature occlusion, motion/structure ambiguity and processing mixed-domain sequences.

\section{References}

[1] H. Longuet-Higgins, "A computer algorithm for reconstructing a scene from two projections," Nature 293, pp. 133-135, September 1981.

[2] O. Faugeras, Three-Dimensional Computer Vision: A Geometric Viewpoint, MIT Press, 1993.

[3] J. Oliensis, "A critique of structure from motion algorithms," Tech. Rep. www.neci.nj.com/ homepages /oliensisi /poleiccv.ps, NEC Research Institute, Princeton, NJ, July 2000.

[4] S. Soatto and R. Brockett, "Optimal structure from motion: Local ambiguities and global estimates," in IEEE Computer Vision and Pattern Recognition, Santa Barbara, CA, pp. 282-288, 1998.

[5] D. Forsyth, S. loffe, and J. Haddon, "Bayesian structure from motion," in International Conference on Computer Vision, Corfu, Greece, pp. 660-665, 1999.

[6] T. J. Broida, S. Chandrashekhar, and R. Chellappa, "Recursive estimation of 3-d kinematics and structure from a noisy monocular image sequence," IEEE Trans. on Aerospace and Electronic Systems 26, pp. 639-656, 1990.

[7] A. Azarbayejani and A. Pentland, "Recursive estimation of motoin, structure, and focal length," IEEE Trans. on Pattern Analysis and Machine Intelligence 17, pp. 562-575, 1995.

[8] J. MacCormick and A. Blake, "A probabilistic exclusion principle for tracking multiple objects," International Journal of Computer Vision 39, pp. 57-71, August 2000.

[9] G. Kitagawa, "Monte carlo filter and smoother for nongaussian nonlinear state space models," Journal of Computational and Graphical Statistics 5(1), pp. 1-25, 1996.

[10] N. Gordon, D. Salmon, and A. Smith, "Novel approach to non-linear/non-gaussian bayesian state estimation," IEE Proceedings 140, pp. 107-113, 1993.

[11] J. S. Liu and R. Chen, "Sequential monte carlo methods for dynamic systems," J. Amer. Statist. Assoc. 93, pp. 1032 $1044,1998$.

[12] C. Tomasi and T. Kanade, "Shape and motion from image streams under orthography: A factorization method," International Journal of Computer Vision 9, pp. 137-154, November 1992.

[13] G. Young and R. Chellappa, "Statistical analysis of inherent ambiguities in recovering 3-d motion from a noisy flow field," IEEE Trans. on Pattern Analysis and Machine Intelligence 14, pp. 995-1013, October 1992.

[14] G. Qian, Q. Zheng, and R. Chellappa, "Reduction of inherent ambiguities in structure from motion problem using inertial data," in IEEE International Conference on Image Processing, Vancouver, BC, Canada, p. MA07.01, 2000.

[15] C. Tomasi and J. Shi, "Good features to track," in IEEE Computer Vision and Pattern Recognition, Seattle, WA, pp. 593$600,1994$. 